\title{
Evaluation of V2X Antenna Performance Using a Multipath Simulation Tool
}

\author{
Edith Condo Neira ${ }^{1}$, Ulf Carlberg ${ }^{1}$, Jan Carlsson ${ }^{1,2}$, Kristian Karlsson ${ }^{1}$, Erik G. Ström ${ }^{2}$ \\ ${ }^{1}$ SP Technical Research Institute of Sweden, Borås, Sweden, edith.condoneira@sp.se \\ ${ }^{2}$ Chalmers University of Technology, Gothenburg, Sweden
}

\begin{abstract}
Antennas are one of the key components for efficient and reliable vehicular communications systems; especially for safety related applications the antenna performance is crucial. It is therefore important to be able to evaluate the antenna performance under realistic conditions so that the best antenna solution can be selected. In this paper we present a statistical method for evaluating the antenna performance by using the antennas radiation patterns and a multipath simulation tool. The multipath environment is generated by a number of incident waves with a specific angle of arrival distribution, defined by the user. In order to generate voltage samples at the antenna ports many sets of incident waves are generated. This simulates a changing environment as, e.g., when the vehicle is moving. By studying the cumulative distribution functions (CDFs) for the voltage samples we are able to compare the performance for different antenna types, positions on the vehicle, etc. The method is fast and effective and, when applied to typical vehicular propagation environments, the importance of having antennas with radiation patterns capable of covering the whole azimuth plane is demonstrated.
\end{abstract}

Index Terms-V2X Antennas; Measurements; Antenna Performance

\section{INTRODUCTION}

In the last few years the interest in vehicle-to-vehicle and vehicle-to-infrastructure communication (together commonly referred to as V2X) has increased significantly. This is mainly due to the many areas of application, such as traffic safety, traffic management and infotainment. In Europe, V2X communications is standardized by ETSI as the ITS-G5 standard [1]. This standard describes a number of safety applications, which require a reliable communication link. In order to achieve such reliable link, antennas with suitable radiation patterns need to be used. The assigned frequency in Europe for vehicle communications is $5.9 \mathrm{GHz}$ and at this high frequency the radiation pattern of an antenna mounted on a vehicle is very complicated with large variations over the solid angle. Thus, it is very difficult to judge which antenna is the best simply by inspecting the radiation pattern.

Many studies have been done in the field of antennas for wireless communications [2]. One example is mobile communications, which in [3], is defined as communication between a fixed base station and a moving user. The base station is normally elevated and the mobile terminal might have any orientation. In this case it is, from the mobile terminal perspective, motivated to study the two extreme environments random line-of-sight (RLOS) and rich isotropic multipath (RIMP) as was done in [4]. The RLOS is defined as only one incident wave on the terminal, but in order to take the user statistics into account this wave has an arbitrary incidence direction and polarization. In RLOS, we can evaluate the antenna performance by studying the cumulative distribution function (CDF) for the voltage samples at the antenna port that is obtained for many incident waves, i.e., many realizations. In the RIMP environment, which is characterized by many incident waves where the angle-of-arrivals (AoA) of the waves are uniformly distributed over all directions in 3D space, the only antenna parameter of importance is the total radiation efficiency, i.e., the radiation efficiency including the mismatch. In RIMP the CDF for the voltage samples at the antenna port will be Rayleigh distributed independent of the radiation pattern.

For V2X communications on the other hand neither RLOS nor RIMP are relevant. For this case the transmitting as well as the receiving antenna is mounted on roughly the same height and scatterers responsible for the multipath are located mostly in the horizontal plane. This means that waves incident on the receiving antenna will come from arbitrary directions in azimuth but will have a limited angular spread in elevation. For this case, we can talk about richness in the sense that we might have different number of incident waves at a given time or position that together gives the voltage sample at the antenna port. In rural areas and when the two communicating antennas are closely spaced there will often be a dominating line-of-sight (LOS) component and the number of non-line-of-sight components (NLOS) will be few. We simulate this by defining few incident waves when computing the voltage samples. In other types of environments, such as urban or when we have many scattering objects in the vicinity (e.g., in a traffic jam) the number of incident waves might be considerably higher.

Unlike to the situation in RIMP the antenna gain pattern will be important for the overall performance in a typical vehicular communication environment. The question is how to determine which antenna is the best. As is shown in Fig. 1 the radiation patterns for two different $5.9 \mathrm{GHz}$ antennas mounted at the same position on a vehicle are very complicated and it is difficult to judge which one is the best. Thus, it is important to have a good methodology for evaluating the performance of high frequency vehicle antennas that are mounted in different positions and used in different road environments. 


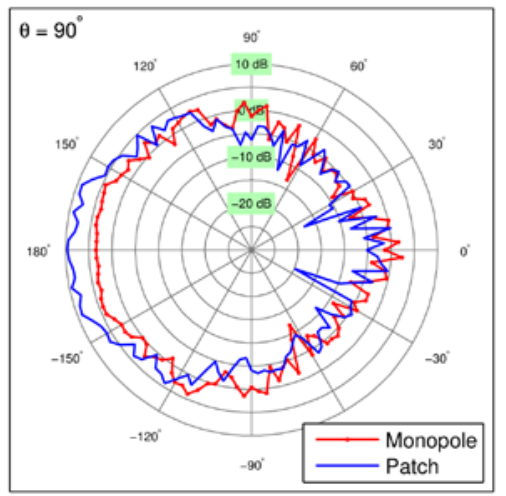

Figure 1. Comparison between the simulated radiation patterns of a quarterwave monopole and a probe-feed patch antenna mounted on the vehicle's windscreen at $5.9 \mathrm{GHz}$ (elevation plane $\theta=90^{\circ}$ ). The front of the vehicle is oriented towards the $180^{\circ}$ direction (negative $\mathrm{x}$-axis).

A number of measurement campaigns have been carried out for V2X communications. Some of them have focused on evaluating different positions in which an antenna could be placed [5-7], and others on the evaluation of the channel for different road traffic environments [8]-[9]. The performance considerations of $\mathrm{V} 2 \mathrm{~V}$ antennas mounted on a vehicle's roof and integrated in an antenna module including other antennas have been evaluated in [10], and [11]. All of these measurements have focused on some specific antenna positions or specific environments. In this paper we focus on the performance of antennas for $5.9 \mathrm{GHz}$ and consider both aspects at the same time, the antenna placement on the vehicle and the road traffic environments.

In this paper we present a method for evaluating the performance of V2X antennas mounted at different positions on a vehicle, and used in different road traffic environments. The method is based on simulations in VIRM-Lab, a computer code which generates a statistically fading environment by a number of statistically varying incoming plane waves [12]. By generating a large number of realizations, each consisting of a number of incident waves on the antenna, the CDF for the voltage at the antenna port is calculated. By comparing the CDFs for different antenna types or positions we are able to determine which configuration will have the best system performance. We simulate different road traffic environments by defining the AoA distribution and the number of waves in each realization.

The paper is organized as follows: In Section II the evaluation method is described and simulations for a few basic antennas are presented. Results for practical antennas mounted on a vehicle are presented in Section III. These results are obtained from simulated as well as measured antenna radiation patterns. Finally, the conclusions are given in Section IV.

\section{ANTENNA EVALUATION METHOD}

The evaluation of the antenna performance is done by using the antenna radiation patterns and by simulating the multipath environment. Due to the relative low height in which V2X transmitting and receiving antennas are mounted, multipath phenomena such as scattering, reflections from surrounding buildings and diffraction from other vehicles are expected [13].
As mentioned before, all of these phenomena are located mostly in the horizontal plane. Thus, the incident waves will come from arbitrary directions in azimuth but within a limited range of angles in elevation. Taking this into account and considering the multipath propagation for different road environments, we define the number of incident waves for different cases. For example, for rural areas where there will often be a dominant LOS component and few reflected components [7], the simulation is done by defining few incident waves. For environments such as urban, suburban or highway where, there will be more multipath components, a higher number of incident waves are considered in the simulations.

For the simulations, we generate the multipath environment by a number of incident waves, defined by their AoA and polarization. To simulate a changing environment as it is in reality; a large number of realizations are generated, each consisting of a number of statistically distributed incident plane waves, which are distributed statistically on the radiation patterns of the receiving antennas. These incident waves generate a voltage at the antenna ports and then, the CDFs of the received voltages at the antenna ports are calculated. It should be pointed out that the AoA as well as the polarization of the incident waves change for each realization, generating a statistically changing fading signal at the antenna port.

\section{A. Definition of Weighted Environment for Vehicular Communications}

As pointed out before, neither RIMP nor RLOS are typical environments for V2X communications. Therefore, we define the environment for $\mathrm{V} 2 \mathrm{X}$ communications as a weighted environment. In the weighted environment the incident waves will come from arbitrary directions in the horizontal plane but within a limited range of angles in elevation. Each wave is linearly polarized with arbitrary polarization. For this environment the CDFs will follow the theoretical Rayleigh as the number of incident waves, $M$, increases. However, the CDFs will be shifted to a lower or higher received power level depending on the radiation pattern, showing the dependency of the directivity of the antenna. Notice that the incident waves have random amplitude and phase, being none of them dominant.

TABLE I. Studied Cases for Weighted Environments

\begin{tabular}{|l|c|c|}
\hline \multirow{2}{*}{$\begin{array}{c}\text { Studied } \\
\text { Case }\end{array}$} & Azimuth & Angles \\
\cline { 2 - 3 } & $0^{\circ} \leq \phi \leq 360^{\circ}$ & $-5^{\circ} \leq \theta \leq 15^{\circ}$ \\
\hline $\begin{array}{l}\text { Case I } \\
\text { (uniform) }\end{array}$ & $-45^{\circ} \leq \phi \leq 45^{\circ}$ & $-5^{\circ} \leq \theta \leq 15^{\circ}$ \\
\hline $\begin{array}{l}\text { Case II } \\
\text { (back) }\end{array}$ & $-135^{\circ} \leq \phi \leq 225^{\circ}$ & $-5^{\circ} \leq \theta \leq 15^{\circ}$ \\
\hline $\begin{array}{l}\text { Case III } \\
\text { (front) }\end{array}$ & & \\
\hline
\end{tabular}

Three different environments have been studied, see Table I. Road environments like highway or urban might be represented by Case I. This case represents an environment in which the incident waves are coming uniformly distributed in the azimuth plane with a certain angle of elevation distribution. Case II and Case III; represent an environment where the waves are incident on either the front $\left(\phi=180^{\circ}\right)$ or the rear $(\phi$ 
$=0^{\circ}$ ) of the vehicle defined by a $90^{\circ}$ sector in azimuth. This might be the case in a rural environment. For all cases, the incident waves are uniformly distributed in azimuth as well as in elevation in the intervals specified in Table I. When computing the CDFs, we have used $10^{5}$ realizations. Each realization contains one and twenty linearly polarized incident waves with random polarization, respectively. The AoA as well as the polarization of each incident wave are statistically independent. The phase of the incident waves are uniformly distributed between 0 and $2 \pi$ radians and the amplitude is Rayleigh distributed. In the figures to follow, the average power has been normalized to one.

\section{B. Performance for Basic Antennas in Weighted Environment}

Three basic $5.9 \mathrm{GHz}$ antennas, a dipole, a quarter-wave monopole centrically mounted on a ground plane with size of 1 $\mathrm{x} 1 \mathrm{~m}^{2}$, and a patch antenna, have been evaluated. For the evaluation, calculated antenna radiation patterns in free space have been used. As can be seen in Fig. 2-4, the CDFs for the dipole antenna show pretty much the same results for the three studied cases. For the quarter-wave monopole antenna, the CDFs for the three studied cases are also very similar. This is because the monopole antenna has a similar radiation pattern compared to the dipole in the range of angles defined by the environments.

The patch antenna is probe-feed and has a length of $11 \mathrm{~mm}$, width of $15 \mathrm{~mm}$ and a ground plane size of $20 \times 24 \mathrm{~mm}$. The substrate is FR4 with a thickness of $1.6 \mathrm{~mm}$. Since the patch is a directional antenna, the CDF changes according to the environment in which it is simulated. In Fig. 3, the incident waves are coming from the opposite direction compared to the patch main beam; this will of course have a negative effect on the performance. However, when the incident waves are coming from the same direction as the patch main beam, the antenna will perform much better than the dipole and the monopole antenna, see Fig. 4.

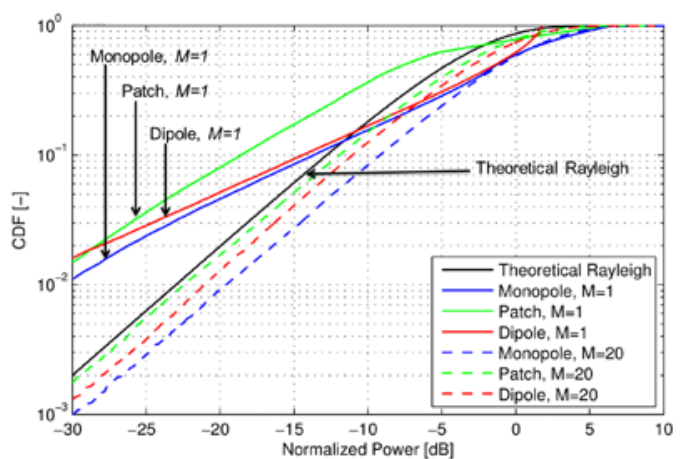

Figure 2. CDFs for linear polarized incident waves. Solid lines represent a weighted RLOS environment $(M=1)$ and dashed lines represent a weighted environment $(M=20)$. The simulations are done for Case I (see Table I). The Patch antenna is radiating towards the negative $\mathrm{x}$-axis.

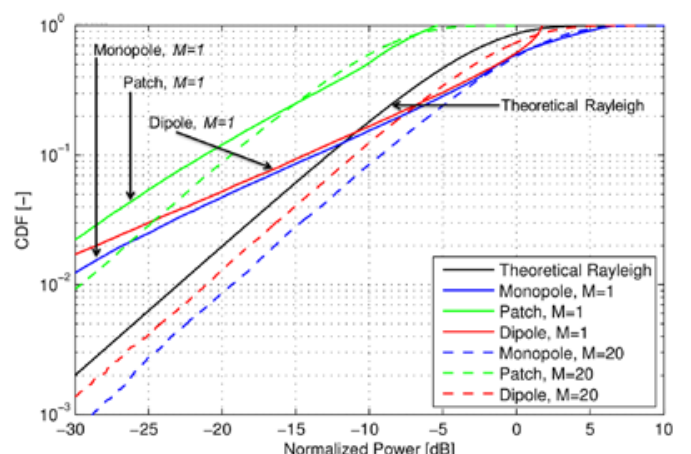

Figure 3. CDFs for linear polarized incident waves. Solid lines represent a weighted RLOS environment $(M=1)$ and dashed lines represent a weighted environment $(M=20)$. The simulations are done for Case II (see Table I). The Patch antenna is radiating towards the negative $\mathrm{x}$-axis. The opposite direction in which the incident waves are coming.



Figure 4. CDFs for linear polarized incident waves. Solid lines represent a weighted RLOS environment $(M=1)$ and dashed lines represent a weighted environment $(M=20)$. The simulations are done for Case III (see Table I). The Patch antenna is radiating towards the negative $\mathrm{x}$-axis. The same direction in which the incident waves are coming.

\section{RESULTS FOR PRACTICAL ANTENNAS}

A few basic antennas were manufactured and the radiation patterns for the antennas mounted on a vehicle in a few different positions were both simulated and measured. The simulations were done in CST Microwave Studio and the measurements were done in a semi-anechoic chamber. For both simulations and measurements, the antennas were mounted on two different positions on the vehicle, the windscreen and the rear window, respectively. The vehicle was aligned along the $\mathrm{x}$-axis with the front pointing in the negative $\mathrm{x}$-axis direction, as shown in Fig. 5. The practical antennas were a quarter-wave monopole and a probe-feed patch antenna, both of them resonating at $5.9 \mathrm{GHz}$. The monopole was mounted in the center of a ground plane with size of $30 \times 30 \mathrm{~mm}^{2}$. The dimensions of the patch antenna were the same as defined in the previous section.

In this section, we have only used vertically polarized incident waves since the two manufactured antennas were vertically polarized. The CDFs are generated from the simulated and measured antenna radiation patterns and a defined weighted environment. For all cases, the CDFs were computed with $10^{5}$ realizations. Each realization contains one and twenty vertically polarized incident waves, respectively. 




Figure 5. Orientation of the vehicle. The antennas are mounted in two different positions; (1) Windscreen and (2) rear window.

\section{A. Performance for Simulated Antennas}

The CDFs for the monopole and patch antennas are shown in Fig. 6. Both antennas are mounted on the windscreen of the vehicle and the vertically polarized incident waves are coming as defined by Case I (see Table I). In this case, we can see that the patch antenna performs better than the monopole when we have twenty vertical polarized incident waves in each realization. However, when we only have one wave in each realization the monopole antenna performs better, except at high levels where the patch becomes better again. This behavior can be understood by analyzing the radiation patterns shown in Fig. 1 where it can be seen that the monopole has a smoother radiation pattern in azimuth and the patch has a higher directivity as well as deeper dips in the pattern.

In Fig. 7, the vertical incident waves are coming as defined by Case III (see Table I). As can be seen, the patch antenna performs much better than the monopole in this case. This is because the incident waves are coming from the same direction in which the patch is radiating, i.e., toward the front of the vehicle. However, when the vertical incident waves are coming from the opposite side in which the patch is radiating, i.e., incident waves on the rear of the vehicle, the monopole performs better than the patch. By comparing Fig. 7 and Fig. 8 we can as expected see that when the antennas are mounted on the windscreen they both perform much better when the waves are incident on the front of the vehicle as compared to the rear. We can also note that the difference between the two antenna types is large when the waves are incident on the front while the difference is smaller when the waves are incident on the rear of the vehicle.

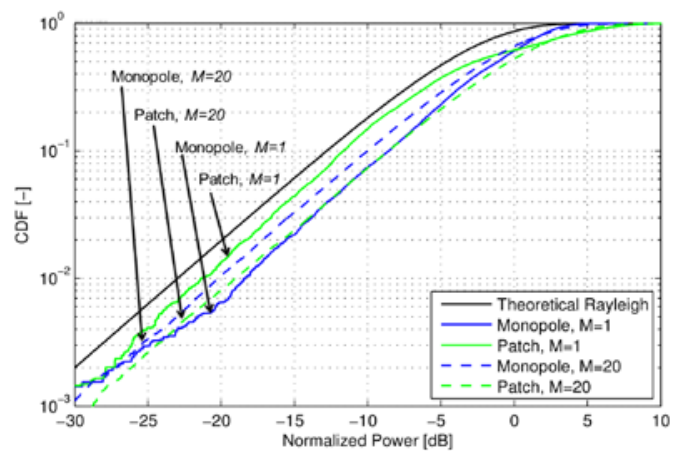

Figure 6. CDFs for vertical polarized incident waves. Solid lines represent a weighted RLOS environment $(M=1)$ and dashed lines represent a weighted environment $(M=20)$. The simulations are done for Case I (see Table I). The Patch antenna is radiating towards the negative $\mathrm{x}$-axis. The antennas are mounted on the vehicle's windscreen.



Figure 7. CDFs for vertical polarized incident waves. Solid lines represent a weighted RLOS environment $(M=1)$ and dashed lines represent a weighted environment $(M=20)$. The simulations are done for Case III (see Table I). The Patch antenna is radiating in the direction in which the incident waves are coming. The antennas are mounted on the vehicle's windscreen.

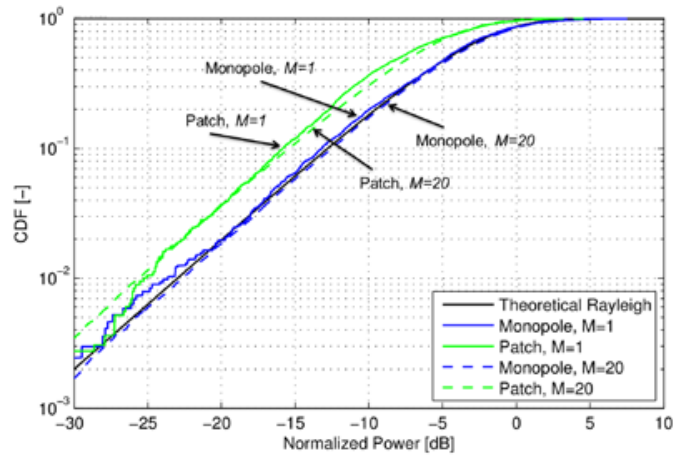

Figure 8. CDFs for vertical polarized incident waves. Solid lines represent weighted RLOS environment $(M=1)$ and dashed lines represent a weighted environment $(M=20)$. The simulations are done for Case II (see Table I). The Patch antenna is radiating in the opposite direction in which the incident waves are coming. The antennas are mounted on the vehicle's windscreen.

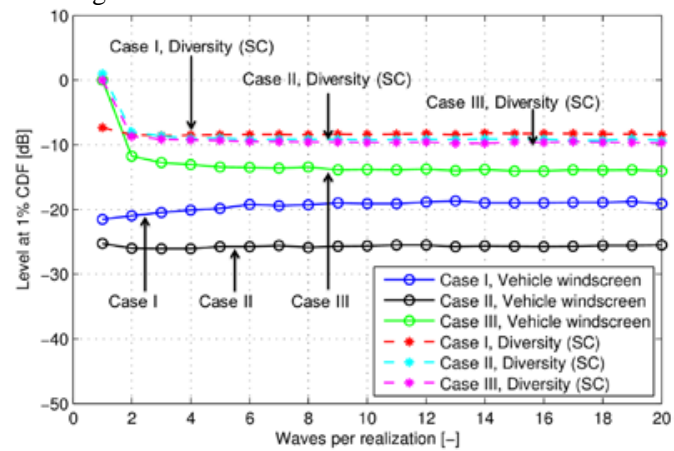

Figure 9. Received power at $1 \%$ CDF-level as a function of vertical polarized incident plane waves. The simulations are done for the three studied cases (see Table I) when the patch is mounted on the vehicle's windscreen. The graph also shows the diversity combined results for the three studied cases when one patch is mounted on the vehicle's windscreen and another one on the rear window.

In Fig. 9, we can see the difference between the three studied cases (see Table I) when the patch antenna is mounted on the windscreen of the vehicle. We can see that the patch performs best in Case III and this is because the vertical incident waves are coming from the same direction in which the patch is radiating, i.e., toward the front of the vehicle. With this knowledge, we can use diversity to improve signal quality. This is also shown in Fig. 9, where selection combining (SC) was used to combine signals from patch antennas mounted on the vehicle's windscreen and rear window, respectively. 


\section{B. Performance for Measured Antennas}

The radiation pattern measurements were done in a semianechoic chamber which had a solid metallic floor temporary filled with absorbers. The probe-feed patch antennas were mounted on the vehicle's windscreen and on the rear window. To perform the measurements, a turntable was used to collect the data every two degrees in the azimuth plane (full $360^{\circ}$ ), and a mast to collect the measurement data for few angles in the elevation plane.

In Fig. 10, the CDFs results for the antenna mounted on both positions are shown. The incident waves are coming as in Case I (see Table I). As can be seen, when we have twenty vertical polarized incident waves, the patch mounted on the vehicle's windscreen performs better than when mounted on the vehicle's rear window.

\section{Comparison Between Simulated and Measured Antennas}

In order to compare simulations with measurements, we have studied the patch antenna mounted on the vehicle's windscreen. The comparison was done for Case I (see Table I). In Fig. 11, it can be seen that the CDFs results for both the simulated and the measured radiation patterns follow the theoretical Rayleigh when we have twenty vertical incident waves. However, the measured radiation pattern is slightly better than the simulated.

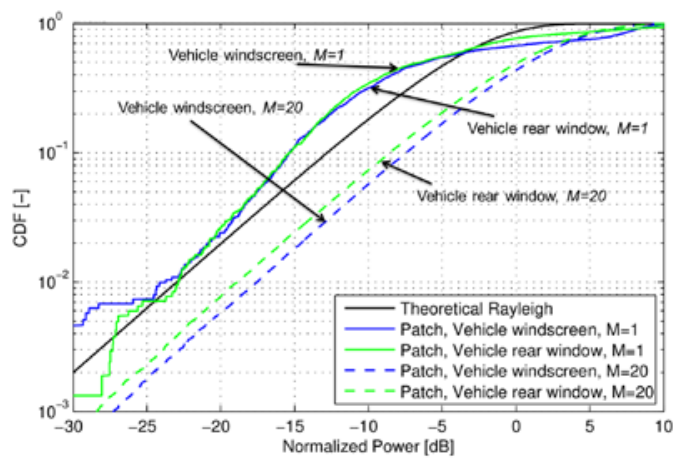

Figure 10. CDFs for vertical polarized incident waves. Solid lines represent a weighted RLOS environment $(M=1)$ and dashed lines represent a weighted environment $(M=20)$. The simulations are done for Case I (see Table I). The patch is mounted in the vehicle's windscreen and rear window.

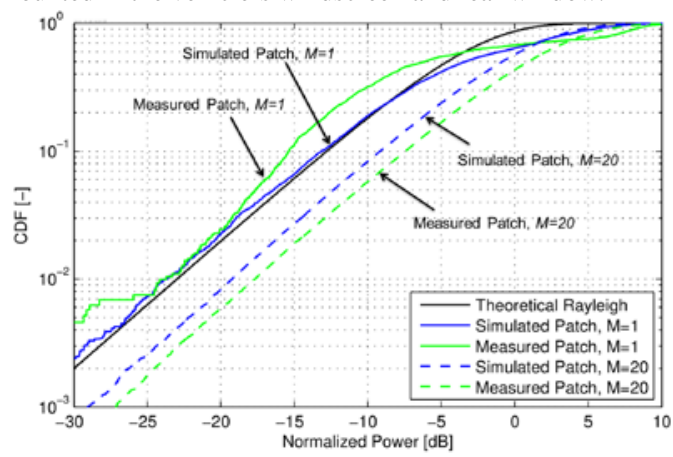

Figure 11. CDFs for vertical polarized incident waves. Solid lines represent RLOS environment $(M=1)$ and dashed lines represent a weighted environment $(M=20)$. The simulations are done for Case I (see Table I)

\section{CONCLUSION}

We have presented a method for evaluating the performance of V2X antennas mounted at different positions and used in different road traffic environments. Simulations and measurement results performed on the vehicle emphasize the importance of antennas with radiation patterns covering the whole azimuth plane. The statistical method that we have presented is fast, effective and in general give us a good idea of a suitable antenna placement on vehicle, the type and the number of antennas that are needed in a real environment.

\section{ACKNOWLEDGMENT}

This work has been supported by The Swedish Governmental Agency for Innovation Systems (VINNOVA) within the VINN Excellence Center Chase.

\section{REFERENCES}

[1] E. G. Ström, "On Medium Access and Physical Layer Standards for Cooperative Intelligent Transport Systems in Europe," Proceedings of the IEEE, vol. 99, no. 7, pp. 1183-1188, July 2011.

[2] A.M.Yadav, C.J.Panagamuwa, and R.D.Seager, "A Miniature Reconfigurable Printed Monopole Antenna for WLAN/WiMAX and LTE Communication Bands," Loughborough Antennas \& Propagation Conference (LAPC), Loughborough, UK, Nov. 2013.

[3] K. Fujimoto and J. R. James, Mobile Antenna Systems Handbook, Second ed. Norwood, MA, USA: Artech House, 2001.

[4] J. Carlsson, U. Carlberg, and P. S. Kildal, "Diversity Gains in Random Line-Of-Sight and Rich Isotropic Multipath Environment," Loughborough Antennas \& Propagation Conference (LAPC), Loughborough, UK, Nov. 2012.

[5] L. Reichardt, T. Fügen, and T. Zwick, "Influence of Antennas Placement on Car to Car Communications Channel," Antennas and Propagation (EuCAP), Proceedings of the 3rd European Conference, Berlin, Germany, Mar. 2009.

[6] S. Kaul, K. Ramachandran, P. Shankar, S. Oh, M. Gruteser, I. Seskar, and T. Nadeem, "Effect of Antenna Placement and Diversity on Vehicular Network Communications," Sensor, Mesh and Ad Hoc Communications and Networks (SECON) pp. 112-121, June 2007.

[7] T. Abbas, J. Karedal, and F. Tufvesson, "Measurement-Based Analysis: The Effect of Complementary Antennas and Diversity on Vehicle-toVehicle Communication," IEEE Antennas and Wireless Propagation Letters, vol. 12, pp. 309-312, Mar. 2013.

[8] J. Kunisch and J. Pamp, "Wideband Car-to-Car Radio Channel Measurements and Model at $5.9 \mathrm{GHz}$," in IEEE Vehicular Technology Conference. VTC 2008-Fall, Calgary, Canada, Sept. 2008.

[9] R. Protzmann, B. Schünemann, and I. Radusch, "The Influences of Communication Models on the Simulated Efffectiveness of V2X Applications," IEEE Commun. Magazine pp. 149-155, Nov. 2011.

[10] O. Klemp, "Performance Considerations for Automative Antenna Equipment in Vehicle-to-Vehicle Communications," URSI International Symposium on Electromagnetic Theory, pp. 934-937, Aug. 2010.

[11] M. Gallo, S. Bruni, M. Pannozzo, and D. Zamberlan, "Performance Evaluation of C2C antennas on Car Body," Antennas and Propagation (EuCAP) Proceedings of the 7th European Conference, Gothenburg, Sweden, April 2013.

[12] U. Carlberg, J. Carlsson, A. Hussain, and P. S. Kildal, "Ray Based Multipath Simulation Tool for Studying Convergence and Estimating Ergodic Capacity and Diversity Gain for Antennas with Given Far-Field Functions," in 20th Internat. Conf. on Applied Electromagnetics and Communications (ICECom), Dubrovnik, Croatia, Sept. 2010.

[13] B. Gallagher and H. Akatsuka, "Wireless Communications for Vehicle Safety: Radio Link Performance and Wireless Connectivity Methods," IEEE Vehicular Technology Magazine, pp. 4-24, Dec. 2006. 